livraisons

d'Histoire

de l'Architecture

\section{Livraisons de l'histoire de l'architecture}

13 | 2007

Architectures des établissements d'enseignement supérieur

\title{
L'Institut catholique de Paris : politique et architecture sous la troisième république
}

The Catholic University of Paris : some thoughts on policy and architecture in the Third Republic

Das Institut catholique de Paris : Politik und Architektur während der Dritten Republik

\section{Anne Goergeon-Liskenne}

\section{OpenEdition \\ Journals}

Édition électronique

URL : http://journals.openedition.org/lha/400

DOI : $10.4000 /$ lha. 400

ISSN : 1960-5994

Éditeur

Association Livraisons d'histoire de l'architecture - LHA

Édition imprimée

Date de publication : 10 juin 2007

Pagination : 9-22

ISSN : 1627-4970

Référence électronique

Anne Goergeon-Liskenne, "L'Institut catholique de Paris : politique et architecture sous la troisième république », Livraisons de l'histoire de l'architecture [En ligne], 13 | 2007, mis en ligne le 10 juin 2009, consulté le 19 avril 2019. URL : http://journals.openedition.org/lha/400 ; DOI : 10.4000//ha.400

Ce document a été généré automatiquement le 19 avril 2019

Tous droits réservés à l'Association LHA 


\section{L'Institut catholique de Paris : politique et architecture sous la troisième république}

The Catholic University of Paris : some thoughts on policy and architecture in the Third Republic

Das Institut catholique de Paris : Politik und Architektur während der Dritten Republik

\section{Anne Goergeon-Liskenne}

« L'Institut catholique, les vieux monuments ont été la passion de mon existence $»^{1}$ (Gabriel Ruprich-Robert) « On peut dire de tout catholique sincère qu'il est profondément un constructeur $»^{2}$ (Paul Claudel)

Fondée en 1875 à la faveur d'une loi sur la liberté de l'enseignement supérieur, l'université, puis institut catholique, s'est figurée dans le sceau qu'elle a fait graver à sa création: inspiré de celui de l'Université de Paris du Moyen Âge, sa "glorieuse devancière ${ }^{3}$, il représente la Sainte Vierge portant l'Enfant Jésus sous un arceau trilobé s'appuyant sur des colonnettes. En dessous, à droite, sont figurées les armes de la Ville de Paris. À gauche sont représentées les armes de la Papauté, attestant de la soumission de l'Institut au Saint-Siège (ill. 1). 


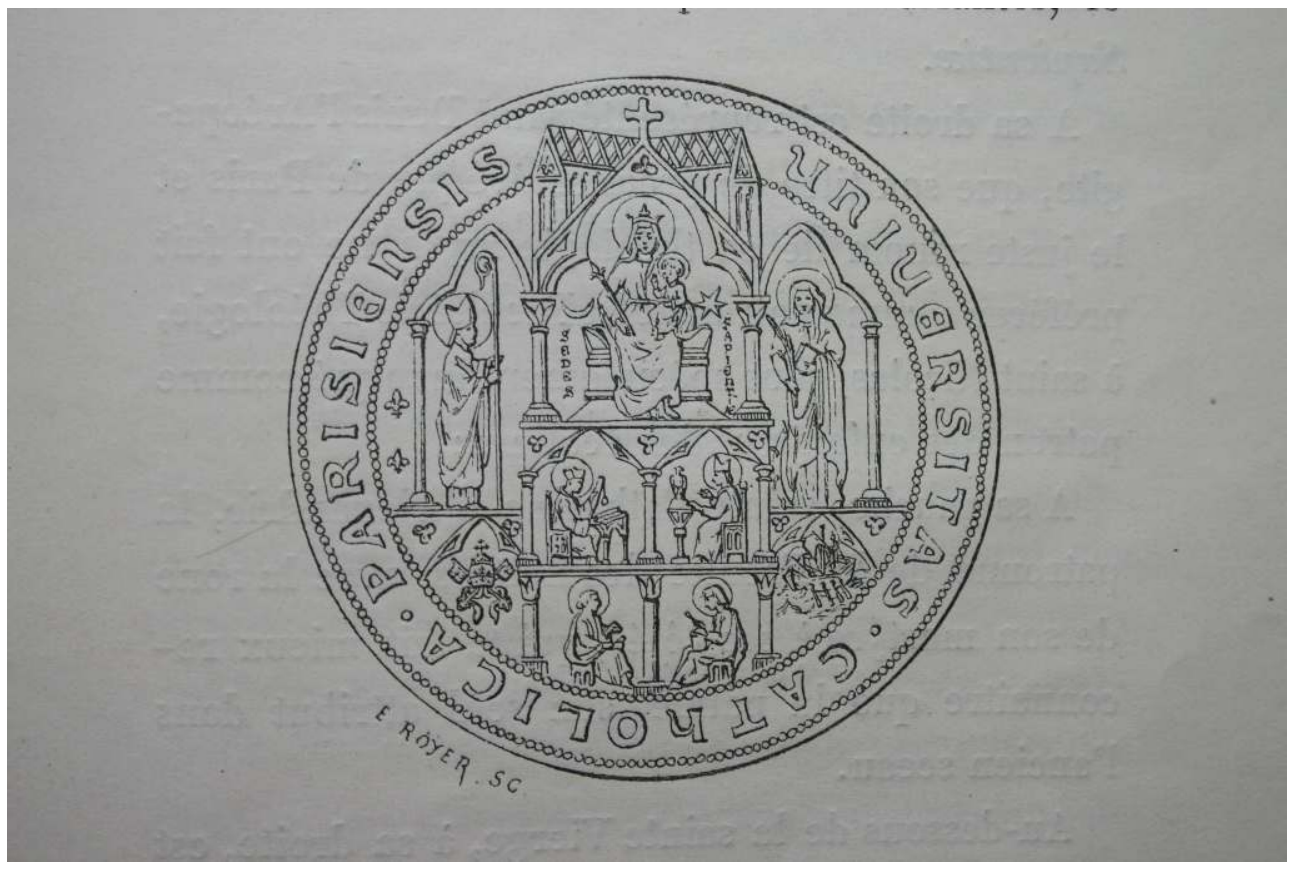

Rapport à son éminence Mgr le cardinal Guibert, archevêque de Paris, sur le choix d'un sceau pour I'Université catholique, 29 mai 1877, p. 8

Cl. A. Georgeon-Liskenne.

2 Vingt ans après, l'architecte Gabriel Ruprich-Robert parait avoir voulu respecter cette référence au modèle médiéval quand il réalise les premiers travaux en bordure de la rue d'Assas.

3 Sujet d'un article paru en 1999 dans un ouvrage collectif ${ }^{4}$, l'institut catholique de Paris (I.C.P.) n'a pas encore fait l'objet d'une monographie sur ses bâtiments bien que des publications sur son histoire paraissent régulièrement, en particulier à l'occasion des anniversaires de sa fondation. Son étude est rendue plus difficile par l'absence d'archives sur sa construction. Les archives de l'I.C.P. sont en effet très lacunaires ${ }^{5}$, celles de l'archevêché de Paris évoquent ponctuellement la question des travaux et les archives de Paris conservent une collection de permis de construire, également partielle au tournant du siècle. La presse spécialisée contemporaine de l'édifice apporte en revanche une contribution importante à la connaissance du bâtiment et les procès-verbaux des conseils d'administration sont une source essentielle sur les étapes de sa réalisation ${ }^{6}$.

\section{5-1934 : I'histoire du bâtiment, reflet de l'histoire houleuse de l'Institut catholique de Paris}

\section{Le couvent des Carmes}

Le couvent des Carmes déchaussés, prison sanglante sous la Révolution, rendue célèbre par les massacres de septembre 1792, avait été sauvé de la destruction en 1797 par sœur Camille de Soyecourt, carmélite, qui revendit les bâtiments au diocèse de Paris en $1841^{7}$. 
La parcelle correspondant aux jardins fut réduite par les aménagements urbains. La rue de l'Ouest - actuelle rue d'Assas - diminue de moitié le jardin des Carmes en 1798; en 1867 la rue de Rennes ampute de $2950 \mathrm{~m}^{2}$ ce qu'il en reste. La parcelle que le couvent met à la disposition de l'université en 1875 est encore suffisamment vaste pour qu'on y édifie un premier bâtiment en 1894. Le terrain affecté à la construction longe les rues d'Assas et de Vaugirard ; à l'est, il aboutit à l'église Saint-Joseph-des-Carmes, commandée par Marie de Médicis, au nord, aux jardins des Carmes, et aux immeubles de la rue de Rennes.

\section{La loi de 1875 et la création d'une université libre}

5 La loi du 12 juillet 1875 contient deux articles sur lesquels repose la création de l'I.C.P. Dans son titre premier, l'article $1^{\text {er }}$ déclare que "l'enseignement supérieur est libre » et l'article 5 déclare que « quand [les établissements] réuniront trois facultés, ils pourront prendre le nom d'université libre ». Le 8 septembre suivant, les cardinaux et archevêques annoncent la création d'une université libre de Paris et l'ouverture d'une souscription auprès des diocèses, destinée à couvrir les frais de la première installation ${ }^{8}$. Lille et Angers souhaitent également recevoir un tel établissement mais on convient qu'une telle université recevra plus d'argent à Paris, comptera plus de professeurs distingués et accueillera plus d'élèves.

6 Créé pour former la jeunesse selon des valeurs chrétiennes et contrer le modernisme scientifique, l'I.C.P. parvient à ouvrir trois facultés en 1875, en droit, lettres et sciences, minimum requis pour détenir le titre d'«université». En 1876, il absorbe l'école ecclésiastique des Carmes, fondée en 1845, et la transforme en un séminaire universitaire. L'école supérieure de théologie, fondée en 1878, est érigée en 1889 en faculté pontificale. L'objectif principal de créer une faculté de médecine, pour soustraire les étudiants aux maîtres matérialistes, ne sera pourtant jamais atteint faute de moyens suffisants ${ }^{9}$. Il existe déjà néanmoins une faculté des sciences, peu fréquentée, mais bien équipée d'un laboratoire de chimie, de deux salles de collections et d'un amphithéâtre de zoologie indépendant, installé dans les locaux des Carmes.

7 L'abbé d'Hulst, vicaire général de Paris, est nommé secrétaire de la Commission permanente de l'« université libre de Paris», dont le nom devient, en octobre 1875, " université catholique », puis, après la loi de mars 1880, « Institut catholique de Paris » ${ }^{10}$. Cet ancien aumônier des soldats pendant la guerre de 1870, se voue au redressement de la France qui passe, selon lui, par l'apostolat intellectuel. Il est nommé recteur de l'I.C.P. en 1881 et exerce cette fonction jusqu'à sa mort en $1896^{11}$. L'absence de moyens le contraint à suspendre les travaux commencés en 1894 destinés à accroître l'espace laissé dans les bâtiments des Carmes.

\section{La loi de séparation de l'Église et de l'État du 9 décembre 1905}

8 Accueilli aux Carmes à titre gratuit, l'Institut devient ensuite locataire de l'archevêché de Paris. Ses bâtiments sont menacés de revenir à l'état comme bien de mense épiscopale par la loi de $1905^{12}$. La solution adoptée en 1910 le sauve de la fermeture grâce au bail que l'État conclut avec lui et renouvelle tous les trois ans. Cette période de mise sous séquestre empêche les investissements de l'I.C.P. qui ne peut ni construire ni détruire, pendant des années. 
9 À partir de 1924, l'I.C.P. reprend les négociations pour le rachat des immeubles situés 74 rue de Vaugirard et 19 rue d'Assas. Avec l'accord du ministère de l'Intérieur et de celui des Finances, une convention de mitoyenneté est signée le 20 juillet 1924 entre la direction des domaines de la Seine, qui administre le séquestre, et la Ville de Paris, délimitant les deux fonds ${ }^{13}$. Le 29 juillet 1927, l'Institut se rend acquéreur de la totalité des locaux, où il vit depuis 1875, à l'exception de la chapelle qui demeure propriété de la Ville de Paris ${ }^{14}$. Le jubilé de 1925 avait offert le prétexte d'une collecte de dons. L'Institut doit débourser 1930000 francs pour racheter ses propres bâtiments. Un arrêté du ministère des Finances daté de 1910 autorisait en effet l'aliénation de propriétés administrées par la direction des Domaines dans les cas où l'adjudication était reconnue impossible ou inopportune. Le Conseil d'État s'était prononcé contre ce type d'aliénation mais les textes furent interprétés et appliqués dans un sens de conciliation ${ }^{15}$. Les parcelles entourant le couvent des Carmes ayant déjà été bâties, il semblait difficile pour l'État d'en faire un autre usage que celui mis en place par l'I.C.P. Le produit de la vente revient par le décret du 25 octobre 1927 du ministère de l'Intérieur à l'Université de Paris. L'entente du recteur, Mgr Baudrillart, avec le président du Conseil, Raymond Poincaré, et sa femme, a sans doute favorisé ce rachat.

Ce sont donc deux phases - 1894-1897 et 1929-1933 - de la construction des bâtiments de l'I.C.P. qu'il convient d'étudier. De ces deux périodes, on possède quelques plans, les avis du conseil d'administration de l'I.C.P., des lettres de l'architecte, des articles de presse mais ni le programme ni les archives sur l'exécution des travaux n'ont été conservés ou du moins retrouvés (ill. 2).

\section{2 : Plan d'ensemble de l'I.C.P.}

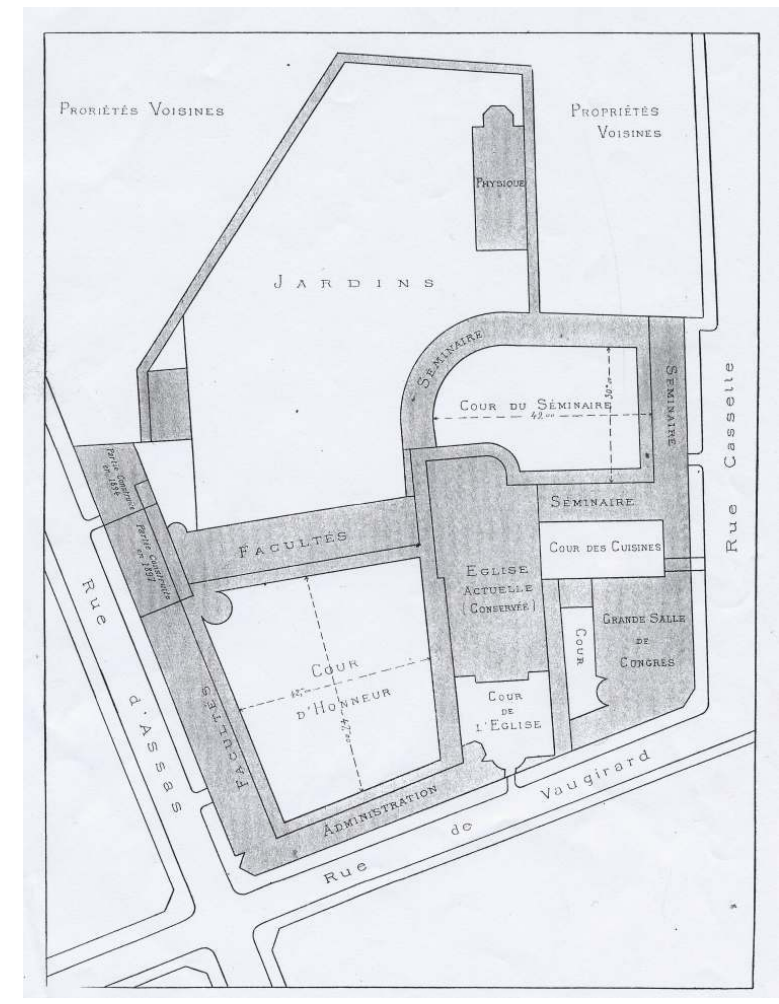

Projet non réalisé de Gabriel Ruprich-Robert, 1898, Mgr P.-L. Péchenard, L'Institut catholique de Paris. 1875-1907, Paris, 1907, 335 p., plan non paginé.

Cl. A. Georgeon-Liskenne. 


\section{Le concours de 1890 : premières réalisations}

\section{L'architecte Gabriel Ruprich-Robert}

En 1894, Mgr d'Hulst charge l'architecte Gabriel Ruprich-Robert ${ }^{16}$ (ill. 3), désigné par la voie d'un concours organisé en 1890, d'amorcer la construction de l'université par un petit bâtiment de deux étages rue d'Assas, côté rue de Rennes.

III. 3 : Détail d'une photographie de famille

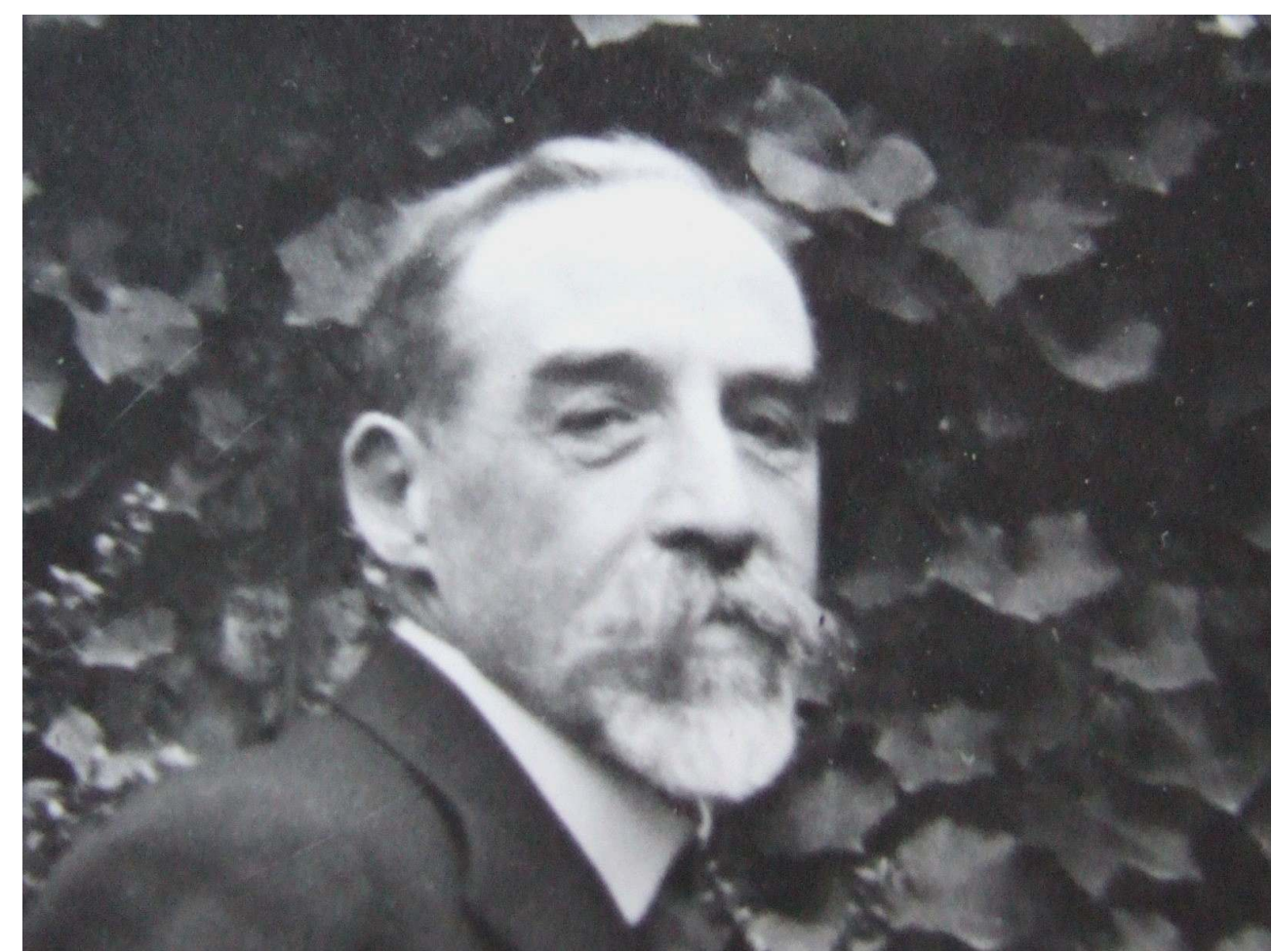

Portrait de l'architecte Gabriel Ruprich-Robert [fin des années 1920]

Cl. M. Marcé

Son père, Victor Ruprich-Robert, avait déjà été chargé de l'entretien et de l'aménagement du couvent des Carmes $^{17}$. Architecte diocésain, il est l'auteur de plusieurs recueils, dont celui sur l'architecture normande aux $\mathrm{XI}^{\mathrm{e}}$ et $\mathrm{XII}^{\mathrm{e}}$ siècles est conservé à la bibliothèque de Fels $^{18}$. L'introduction rend hommage au maître, Viollet-le-Duc, qui « a su poser les bases et développer les principes de l'art du Moyen Âge, [...] [qui] a justifié l'emploi des formes successives de l'architecture $»^{19}$.

L'héritage rationnel et médiéval de ces deux maîtres est à l'œuvre à l'Institut catholique. Gabriel Ruprich-Robert a en outre beaucoup étudié comme architecte en chef des monuments historiques les bâtiments du pays d'Auge en Basse-Normandie et en particulier les pans de bois ${ }^{20}$. De ces études ressort son intérêt pour le décor en brique. Elle «y est en effet montrée dans tous ses états : juste cuite ou polychrome et dans lesquelles n'interviennent que les couleurs des vernis qui forment pour les granges et les étables un large jeu de losanges sur la totalité de leurs façades ou en relief, avec des briques posées de biais [...] $»^{21}$. Elle caractérise la plupart des bâtiments édifiés par 
Ruprich-Robert et particulièrement l'I.C.P. Elle en habille tous les murs de façade, en alternance avec les chaînes et arcs de pierre autour des ouvertures, et forme de discrets motifs décoratifs. Les allèges des baies, divisées_par des meneaux, sont composées de briques vernissées: ces variations «semblent des planches grandeur nature tirées de quelque manuel à l'usage des briqueteurs ${ }^{22}$. À Paris, dans le $\mathrm{VI}^{\mathrm{e}}$ arrondissement où la pierre de taille est de mise, une telle longueur de façade uniquement composée de briques change la tonalité du quartier (ill. 4$)^{23}$.

\section{4 : Institut catholique de Paris, bâtiment de 1894}

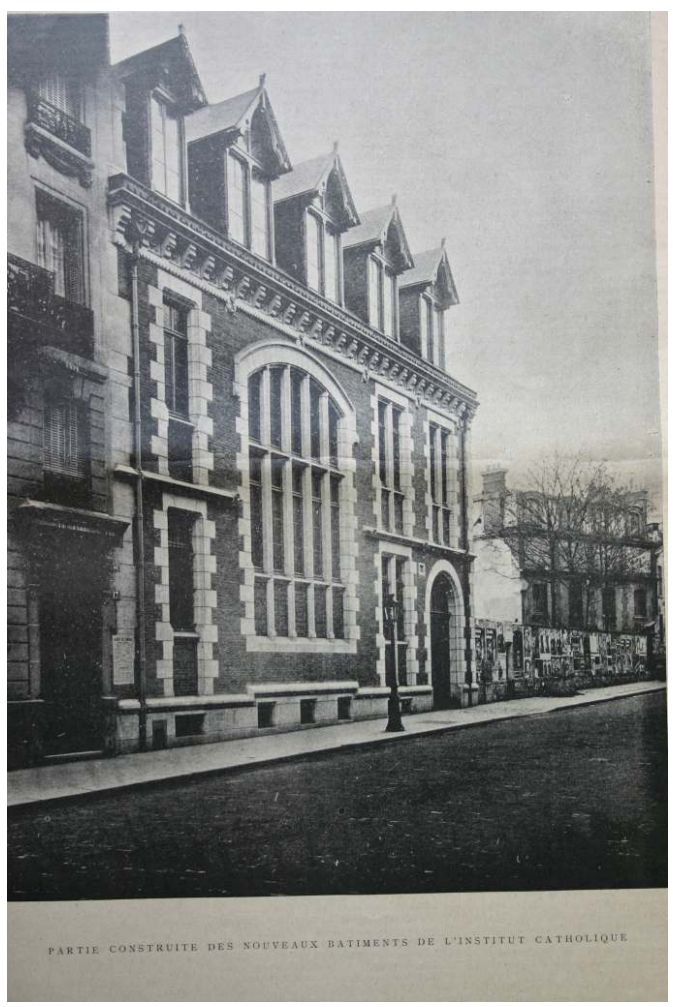

"Partie construite des nouveaux bâtiments de l'Institut catholique », La France illustrée, n 1096, 30 novembre 1895, p. 314-323, p. 321

Cl. A. Georgeon-Liskenne

La structure qui soutient le remplissage de brique et pierre est en « charpente et pans de fer apparents facilement démontables afin de pouvoir modifier les divisions intérieures si le besoin s'en faisait sentir ultérieurement. Le chauffage fonctionne par air pulsé passant dans les allèges et débouchant sous les baies ${ }^{24}$. Louis-Charles Boileau salue en 1900 cette utilisation sincère des matériaux et le souci du détail décoratif (ill. 5) ${ }^{25}$, les motifs floraux des ancres en fer forgé, des rosaces et des gâbles des lucarnes ${ }^{26}$. 
III. 5 : Façade de I'I.C.P. sur la rue et coupe transversale

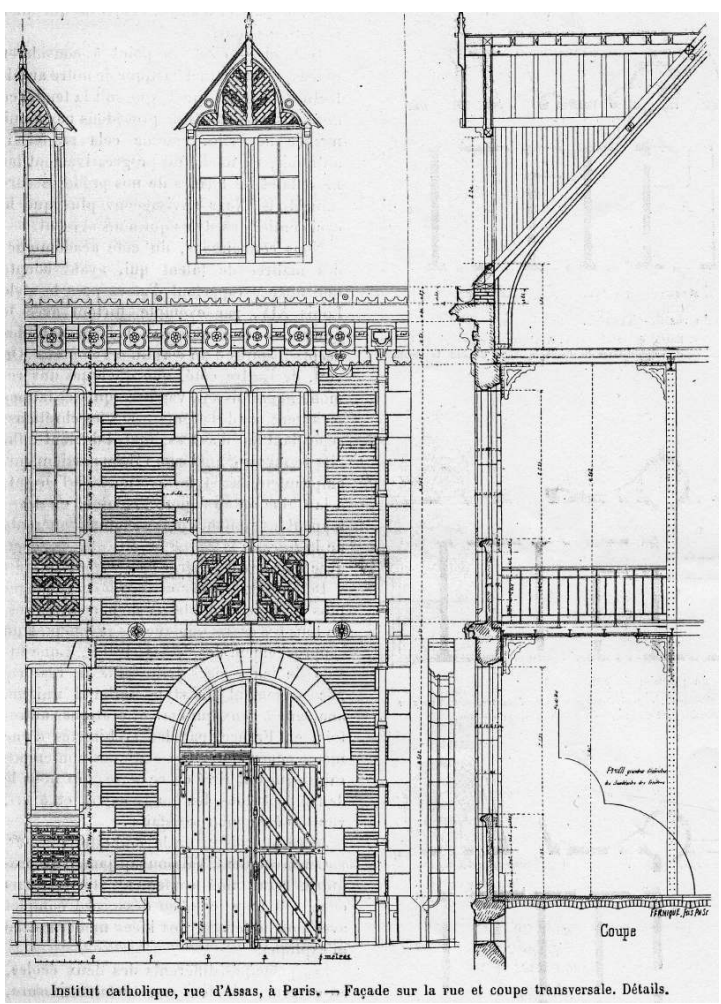

Détails, dessins de Gabriel Ruprich-Robert publiés dans L'Architecture, 1900, p. 200-206, p. 202

Cl. A. Georgeon-Liskenne

Une porte cochère, portant le numéro 19, ouvre, après un passage, sur le rez-de-chaussée. Il donne accès, par deux perrons de pierre, à la salle des conférences s'élevant sur deux étages. Un bâtiment complémentaire, commencé en 1897, est accessible par ce même passage. Les cloisons sur le couloir sont en brique de verre. On accède aux divers étages de ce bâtiment par un escalier en fer et bois, situé dans une tourelle d'inspiration médiévale en brique et pierre. Au dernier niveau est installée la bibliothèque.

En juillet 1898 le conseil d'administration annonce l'affectation de 9000 francs supplémentaires pour adapter le bâtiment de 1894 au nouveau, inauguré en 1898, mais finalement interrompu faute d'argent. Un projet de reconstruction du séminaire daté de novembre 1898 ne verra, pour cette raison, jamais le jour. Il aurait entraîné la destruction de l'ancien couvent, laissant à la place un quadrilatère autour de la chapelle des Carmes ${ }^{27}$.

17 Gabriel Ruprich-Robert continue à s'occuper du bâtiment, comme en témoigne la correspondance retrouvée à l'I.C.P. à propos du chauffage et de divers aménagements ${ }^{28}$. L'architecte, qui avait été désigné très jeune pour édifier la première partie de l'Institut, prend sa retraite au moment où reprend le chantier. Il est cette fois sous la direction d'un maitre d'ouvrage qu'il connait bien, Alfred Baudrillart, recteur de l'Institut.

\section{Le second bâtiment}

Nés tous deux en 1859, ce sont des amis de longue date puisque, à neuf ans, ils fréquentaient l'école Bossuet, située dans les bâtiments des Carmes $^{29}$. Leur 
correspondance et les archives de l'Institut permettent de suivre les étapes de la construction de la seconde partie des bâtiments sur les rues de Vaugirard et d'Assas. Le recteur préférait un emplacement en bordure des rues et non du jardin "pour qu'ils soient très visibles et que le public s'y intéresse ${ }^{30}$. Ils décidèrent aussi de les faire élever jusqu'à la hauteur maximale autorisée ${ }^{31}$. Dans une lettre-programme du 10 février 1929, Gabriel Ruprich-Robert, répondant à une note du recteur du 7 février, indique ses priorités : il faut édifier en premier le bâtiment donnant sur la rue de Vaugirard, pour abriter le logement du concierge et la maison des étudiantes. Doivent y être aussi aménagés des espaces pour le secrétariat, l'école supérieure des sciences économiques et commerciales (E.S.S.E.C.) ${ }^{32}$ et les autres services comme ceux de la chimie, la bibliothèque. La seconde tranche relierait le nouveau bâtiment avec celui construit en 1897. Il comporterait une entrée, une tour intérieure pour les magasins de livres et une salle de lecture, un grand escalier, un préau, huit salles de cour, un laboratoire de physique ${ }^{33}$. «Le dernier chantier devait combler l'espace de 55 mètres qui séparait les constructions précédentes $»^{34}$. L'assemblée générale de l'I.C.P., réunie en novembre 1930, adopte le principe d'une nouvelle tranche de constructions destinée à réunir les deux bâtiments et prévoit une dépense de trois millions et demi de francs, y compris l'aménagement de la bibliothèque ${ }^{35}$. Pour financer les travaux, elle lance une nouvelle souscription qui, en avril 1931, atteint 1914279 francs. Un prêt négocié auprès du Crédit industriel et du Comptoir d'escompte, et le loyer versé par l'E.S.S.E.C., complètent le dispositif.

À la charpente métallique succède l'ossature en béton armé. Le 5 août 1929, le conseil d'administration presse l'architecte de s'adjoindre un technicien du ciment armé. L'ingénieur des Arts et manufactures Jean Braive est choisi. Une lettre est révélatrice des difficultés rencontrées par l'architecte dès les débuts de ce nouveau chantier :

En ton absence [celle de son ami Baudrillart] on a piétiné lamentablement [...]. À cela

plusieurs causes: l'envoi de matériaux défectueux, que je ne pouvais cependant pas admettre et le retard dans la fourniture de la pierre du côté de la cour qu'il a été très difficile d'obtenir en carrière. [...] La question, si complexe, du réassortiment des pierres et des briques, est réglée depuis longtemps et, dans le cahier des charges, il est prévu des pénalités ${ }^{36}$

Le parti architectural reste le même, en référence à l'architecture de la fin du $\mathrm{XV}^{\mathrm{e}}$ siècle et du premier âge de la Renaissance française. L'angle des rues de Vaugirard et d'Assas est inauguré en novembre $1930^{37}$. L'architecte a fait le choix du pan coupé avec trois niveaux d'encorbellement. Il a légèrement simplifié la mouluration extérieure, fait apparaître des parois en briques jaunes dans les parties couvertes, et a eu recours à la mosaïque pour décorer les piliers à facettes de la galerie sur cour. L'escalier hors œuvre est conçu sur le modèle des tourelles d'escalier de la fin du Moyen Âge. Aux toits d'ardoise supportant de larges lucarnes succède la forme en carène de navire qui permet un gain de surface ${ }^{38}$; la toiture surélevée loge trois étages supplémentaires. La partie sur la rue d'Assas est inaugurée en novembre 1934 mais il manque encore l'installation de la grande salle de la bibliothèque et de quelques salles de cours. À chaque faculté est attribué un amphithéâtre distinct ${ }^{39}$ (ill. 6). 


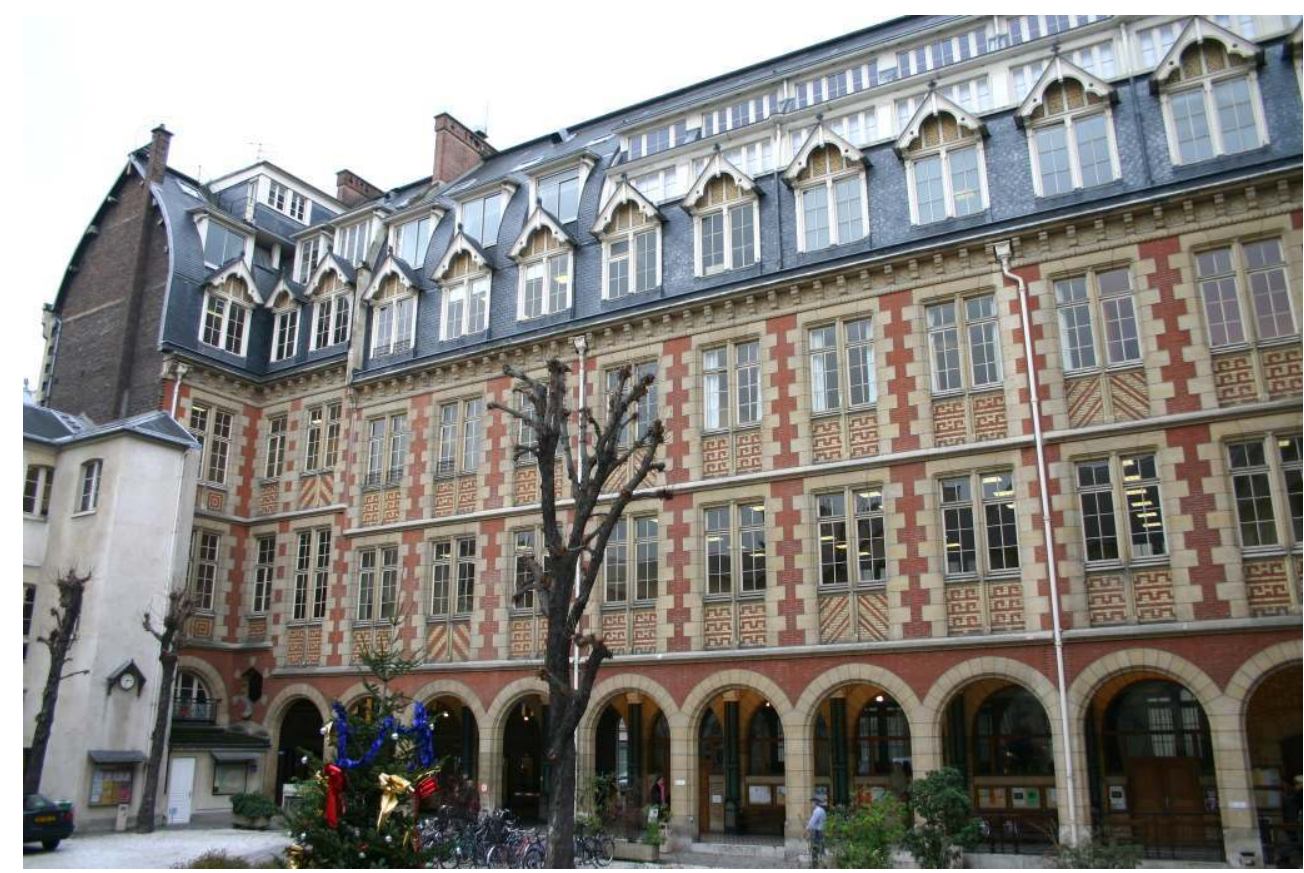

Bâtiments de 1929-30 et 1931-1934

Cl. A. Georgeon-Liskenne

21 La réalisation de Gabriel Ruprich-Robert est saluée en 1935 par la profession. Plusieurs articles paraissent dans la presse spécialisée comme la revue L'Architecture ${ }^{40}$; l'Académie des beaux-arts lui accorde le prix Nicolas Bailly en 1935 et la Société centrale des Architectes y ajoute la première de ses trois grandes médailles de l'architecture privée attribuée pour ses "qualités de composition, sa bonne exécution, sa valeur d'art ${ }^{41}$. L'Architecture cite ce jugement du jury de la SCA :

On retrouve dans tout l'édifice l'esprit du Moyen Âge adapté aux besoins modernes, c'est ce qui lui donne tout son charme. [...] En le parcourant, on y découvre des arrangements imprévus, pittoresques, où les matériaux toujours apparents, remplissent franchement leur rôle $e^{42}$.

Gabriel Ruprich-Robert gardera deux regrets à l'issue de ces chantiers: ses relations houleuses avec le conseil d'administration néanmoins atténuées par son amitié pour Mgr Baudrillart, et l'installation d'un édifice totalement étranger à l'esprit ogival néonormand adopté pour l'I.C.P. : le laboratoire du physicien Édouard Branly, construit au milieu du jardin du séminaire ${ }^{43}$. Un don de 600000 francs avait en effet été offert par François Coty, président du conseil d'administration de la société «Radio-Branly » pour financer un bâtiment à l'abri des trépidations de la circulation qui nuisaient aux expériences électromagnétiques du savant. Les travaux sont menés en 1931 et 1932 sous la direction de son gendre, l'architecte Paul Tournon ${ }^{44}$. Un puits est creusé pour fonder une pile de maçonnerie indépendante et isolée des mouvements du sol, sur laquelle sont posés les appareils du chercheur ${ }^{45}$. Paul Tournon, architecte de l'église du Saint-Esprit, avenue Daumesnil à Paris, édifiée simultanément (1928-1935), ne recourt pourtant pas à la brique dans cet édifice qu'il recouvre d'un simple enduit. Ruprich-Robert s'élève dès 1931 contre ce projet qu'il trouve peu en accord avec l'esthétique du lieu ${ }^{46}$. 

Seconde guerre mondiale à la dynastie Ruprich-Robert - Victor, Gabriel et, selon un procès-verbal du conseil d'administration en 1929 et quelques plans signés, son fils Étienne - ${ }^{47}$.

Par la suite l'immeuble sis 26 rue d'Assas sera loué par la paroisse Saint-Germain-des-Prés à l'I.C.P. Les travaux réalisés en 1974 permettent de lui donner une tonalité décorative proche du bâtiment principal situé en face.

À partir du rachat des parcelles et de la construction de la seconde tranche des bâtiments, l'I.C.P. acquiert sa configuration actuelle. Passé de 315 étudiants en janvier 1878 à 2062 en 1930, il a, malgré ses difficultés, réussi à ouvrir et à entretenir au total six facultés - philosophie, droit, droit canonique, théologie, sciences et lettres-, une école des langues orientales, l'E.S.S.E.C., et l'Institut d'études sociales anciennes ${ }^{48}$. Après s'être rattaché à ses débuts à l'exemple médiéval de l'antique Sorbonne, l'Université de Paris, idéalisée et réinterprétée par son sceau, l'I.C.P. a voulu évoluer vers un modèle plus contemporain, celui des universités anglaises et américaines comme l'vvy League qui offrent à leurs étudiants un domaine universitaire complet, un «lieu de vie intellectuelle $»^{49}$ avec l'église, la maison, le jardin, le séminaire, les amphithéâtres, bibliothèques et laboratoires. Pour accomplir ce programme, les recteurs successifs de l'Institut ont laissé une grande liberté de ton à l'architecte qui a adopté pour son édifice un style rationaliste, égayé de réminiscences néo-normandes et médiévales, donnant à son ouvrage une agréable souplesse formelle.

\section{NOTES}

1. Je tiens à remercier Madame Marie Malinine-Sollogoub, archiviste de l'Institut catholique pour son accueil efficace et chaleureux, et la famille Ruprich-Robert, en particulier la belle-fille de l'architecte, Mme Ruprich-Robert, et M. et Mme Marcé qui m'ont reçue avec confiance et ouvert leur documentation.

Archives de l'Institut catholique (désormais arch. I.C.P.), R.Ba 152, lettre de Gabriel RuprichRobert à Mgr Baudrillart, 10 août 1934.

2. Soixantenaire de l'I.C.P. - 1875-1935, Paris, 1935, 76 p., discours de Paul Claudel, p. 46.

3. Rapport à Son Éminence Mgr le cardinal Guibert, archevêque de Paris, sur le choix d'un sceau pour l'Université catholique, 29 mai 1877, adopté par le conseil supérieur des évêques fondateurs le 25 juillet,1877, arch. de l'I.C.P., F1.

4. Jean-Baptiste Lebigue, «L'Institut catholique de Paris », Universités et grandes écoles à Paris. Les palais de la science, Paris, Action artistique de la Ville de Paris, 1999, 222 p., p. 175-176.

5. Les registres du conseil d'administration, par exemple, n'ont été versés aux archives qu'en février 1976. Le service des archives a été créé en 1975 à l'occasion du centenaire de l'Institut. Sœur Anne-Marie Abel est la première responsable du service.

6. L'Institut est placé dès 1875 sous l'autorité des évêques fondateurs et protecteurs réunis en association en 1922 sous la tutelle de l'archevêque de Paris. Il est administré par un conseil d'administration et dirigé par le recteur. Cette association est reconnue d'utilité publique par la loi du 18 juin 1941, validée par l'ordonnance du 9 août 1944. 
7. L'acte de vente de la propriété est conservé dans les archives de l'I.C.P., fonds des Carmes, Ca 6, art. 1

8. En 1876, l'I.C.P. a collecté 1015000 francs par souscription. Arch. I.C.P., Collection de documents relatifs à la fondation et à l'administration de l'Université catholique de Paris, fonds de l'assemblée générale des évêques fondateurs et protecteurs de l'I.C.P., 1875-1879, procès-verbal de la séance du 29 au 31 mars 1876 du conseil des évêques fondateurs, n.p.

9. L'obstacle majeur évoqué en conseil est celui de l'approvisionnement en cadavres sans lesquels une faculté de médecine ne peut faire travailler ses étudiants. Arch. I.C.P., Collection de documents [...], op. cit.

10. La loi du 18 mars 1880 établit que « les établissements d'enseignement supérieurs [libres] ne pourront en aucun cas prendre le titre d'université ». Elle crée une césure complète entre enseignement public et privé.

11. Mgr Pierre-Louis Péchenard prend la suite de 1896 à 1907. Puis il est évêque de Soissons jusqu'à sa mort en 1920. Le cardinal Alfred Baudrillart né à Paris le 6 janvier 1859, prêtre de l'Oratoire et normalien, lui succède de 1907 à 1942, date de sa mort. Membre de l'Académie française en 1918, évêque titulaire en 1921, archevêque en 1928, cardinal en 1935, il a permis l'extension de l'I.C.P. rue d'Assas dans sa forme actuelle.

12. Le dernier bail est signé avec le bureau des séminaires de l'archevêché de Paris le 13 juillet 1898 pour neuf années qu'interrompt la loi de séparation de 1905.

13. Arch. de l'I.C.P., fonds des Carmes, Ca 8, rapport sur la mitoyenneté entre le domaine séquestre et la Ville de Paris, 1924. La chapelle des Carmes consacrée au culte et ses dépendances sont gérées par la direction des affaires municipales de la Ville de Paris (loi du 13 avril 1908) et le foncier de l'I.C.P. dépend de la direction des domaines de la Seine (liste des terrains et immeubles loués par l'I.C.P. publiée au J.O. le 11/08/1909).

14. Il s'agit des immeubles sis 70 à 74 rue de Vaugirard, 19 à 23 rue d'Assas, 12, 16 et 24 rue Cassette.

15. Les Cahiers de l'impartial, « La vente de l'Institut catholique », 2 juillet 1927, p. 201-202.

16. Gabriel Ruprich-Robert est né à Paris le 5 octobre 1859. Bachelier ès sciences, élève de son père et de l'École des beaux-arts, il devient architecte en chef des monuments historiques en 1887 et inspecteur général en 1925. Il meurt en mars 1953 à l'âge de 94 ans.

17. En 1858 par exemple Victor Ruprich-Robert construit un bâtiment d'un étage en bordure des cloîtres de l'église pour les Dominicains du père Lacordaire. Arch. de l'I.C.P., Ca 8.

18. Victor Ruprich-Robert, L'Architecture normande aux XI et XII siècles en Normandie et en Angleterre, Librairie des imprimeurs réunis, 1889, 2 vol. [Gabriel Ruprich-Robert, éditeur scientifique]. La bibliothèque de Fels est la bibliothèque de l'Institut catholique.

19. Victor Ruprich-Robert est nommé professeur suppléant de Viollet-le-Duc à l'École royale de dessin pour le cours de l'histoire et de la composition de l'ornement en 1843 ; il devient titulaire de l'École, devenue impériale, en 1859.

20. "Études de pans de bois normands ", La Construction moderne, 26 janvier 1889, t. IV, 1888-1889, p. 185-196. L'architecte reçoit la médaille du salon de 1888 en récompense de ses aquarelles de maisons à pans de bois.

21. Aude de Rouffignac, Victor et Gabriel Ruprich-Robert et l'évolution du goût pour les architectures régionales, mémoire de maîtrise en histoire de l'art, Paris IV, 1993, 2 vol. , t. 1, p. 107.

22. Bernard Marrey, Dictionnaire des monuments de Paris, dir. par Jean Colson et Marie-Christine Lauroa, Paris, éd. Hervas, 1992, 917 p., p. 364. Le même principe de construction est repris dans un ensemble d'ateliers situés au 210 rue du faubourg Saint-Antoine en 1905: une ossature métallique est habillée de briques dégageant de grandes baies vitrées. « Le bâtiment vaut par le rapport de creux et de pleins et par le soin apporté aux détails, comme les minces chapiteaux de pierre qui coiffent les piliers de brique et supportent les linteaux métalliques des planchers ", ibid., p. 285. 
23. L'usage de la brique s'est répandu quand sa fabrication s'est industrialisée au milieu du XIXe siècle et son emploi s'est généralisé dans les expositions universelles. Ce matériau est très utilisé dans les programmes industriels - usine Menier à Noisiel, usine élévatrice des eaux, quai de la Rapée, à Paris -, administratifs - mairies -, scolaires - en particulier les lycées édifiés par la Troisième République, comme le lycée Lakanal construit par Anatole de Baudot à Sceaux en $1882-$.

24. Bernard Marrey, La Brique à Paris, Paris, éd. du Pavillon de l'Arsenal, Picard, 1991, 219 p., notice no 100, p. 104.

25. L.- C. Boileau, «Causeries - art et pratique », l'Institut catholique, L'Architecture, samedi 9 juin 1900, no 23, p. 200-206.

26. Victor Ruprich-Robert est l'auteur d'une Flore ornementale de 152 planches publiée en 1866 dans laquelle son fils semble avoir puisé de nombreux motifs.

27. Arch. I.C.P., Ca 8, article 2. Les permis de construire ont été versés de façon lacunaire aux archives de Paris. Celui de l'I.C.P. délivré en février 1894 devrait se trouver dans les dossiers de la cote V.011 141 mais seule a été conservée une autorisation de modifier l'avancement d'une lucarne sur la rue. Les recherches en V.011 142 n'ont pas été plus fructueuses. V.012/25 documente la période 1931-1933 et non la tranche des travaux de 1929-1930. V.013/11 ne contient rien sur la rue d'Assas. Après la Seconde guerre mondiale, il est utile de consulter les cotes 1069 W 43 sur les projets de surélévation de 1962-1963 et 1251 W 62 sur les aménagements sous combles de 1992. En revanche le sommier foncier en DQ18 1299 et le calepin des propriétés bâties en D1P4, carton 47, ne mentionnent pas l'I.C.P.

28. Arch. I.C.P., R.Ba 152.

29. Cette école fut obligée de déménager rapidement en 1875, lors de la création de l'I.C.P.

30. Arch. I.C.P., Ca 1, conseil d'administration du 20 février 1929.

31. «Il n'est pas admissible qu'on construise aux frais de la charité publique des bâtiments d'un ou deux étages dans une ville où le terrain est aussi cher ", archives de l'archevêché de Paris, 5J1, lettre de G. Ruprich-Robert à Mgr Baudrillart, 10 février 1929.

32. L'E.S.S.E.C. est fondée en 1907. Elle s'installe dans les locaux de l'I.C.P. en 1913 et déménage en 1973 à Cergy-Pontoise.

33. Archives de l'archevêché de Paris, 5J1, lettre de G. Ruprich-Robert du 10 février 1929.

34. Jean-Baptiste Lebigue, «L'Institut catholique de Paris », op. cit., p. 176.

35. Arch. I.C.P., Ca 1, séances du conseil d'administration des 5 août 1929 et 18 juin 1931. Un devis nettement moins élevé de 2500000 francs comprenant la maçonnerie, le ciment armé, le chauffage, la charpente, la couverture, la serrurerie, la menuiserie avait été adopté en 1929 puis revu à la hausse un an plus tard, au grand mécontentement du conseil d'administration.

36. Arch. I.C.P., R.Ba 152.

37. La faculté de théologie et l'E.S.S.E.C. étaient dotées de "vastes amphithéâtres dont l'acoustique ne [laissait] rien à désirer ». A. Baudrillart, 25 ans de rectorat. Institut catholique de Paris. 1907-1932, Paris, 1932, 982 p., p. 355.

38. Cette forme répond peut-être aussi à un choix symbolique. Mgr d'Hulst, premier recteur, se plaisait à comparer l'Institut catholique à « un vaisseau de haut bord» et Mgr Baudrillart, son confident et son biographe, reprit en 1925 la même image pour «symboliser la marche en avant sur des flots combien incertains ». A. Baudrillart, Nos grandes écoles. L'Institut catholique, 1930, 130 p., p. 20.

39. Grâce aux plans de 1931 conservés aux Archives de Paris (V.012/25), on connaît la répartition exacte des niveaux : - rez-de-chaussée : préau, vestiaire et amphithéâtre de $115 \mathrm{~m}^{2}$; - 1er étage : amphithéâtre et salle de lecture de $322 \mathrm{~m}^{2} ;-2$ e étage : deux amphithéâtres de $115 \mathrm{~m}^{2}$ et $140 \mathrm{~m}^{2}$, salle d'archives de $63 \mathrm{~m}^{2}$; - 3e étage : deux amphithéâtres et un magasin de livres ; - 4e étage : annexe de la bibliothèque; - le 5 e étage est réparti en deux niveaux de petites salles sous combles. 
40. L'Architecture, 1935, vol. 2, «Rapport du jury de l'architecture privée présenté par M. Jean Hulot», p. 315-325 [en particulier p.316-317, "grande médaille de l'architecture privée » et photographies p. 315-317].

41. Soixantenaire de l'Institut catholique. 1875-1935, Paris, 1935, 76 p., p. 15.

42. L'Architecture, op. cit., p. 317.

43. Édouard Branly (1844-1940) faisait partie du corps des professeurs de l'I.C.P. depuis 1875.

44. En 1936, la construction, dont le coût a dépassé le don initial, n’a toujours pas été payée en totalité et l'I.C.P. doit couvrir une partie des frais à cause du décès de François Coty. Devenu «musée Branly » à la mort du savant, le bâtiment est surélevé en 1963 et accueille l'Institut supérieur d'électronique (arch. de Paris, 1069 W 63).

45. "Un laboratoire pour un physicien, rue de Vaugirard à Paris ", L'Architecte, 1934, p. 145-148, fig. 317-323. Les plans sont conservés aux archives de Paris, V.012/25.

46. " Ce sera la destruction du jardin et de la physionomie du séminaire à laquelle tu tenais au point (excuse-moi) de compliquer la vie de l'Institut catholique [...] au profit d'un savant dont les jours sont comptés » (lettre de Gabriel Ruprich-Robert à Mgr Baudrillart, 27 mars 1931, arch. de l'I.C.P., R.Ba 152).

47. Étienne Ruprich-Robert est né en 1890. Il est l'un des neuf enfants de l'architecte. La correspondance conservée à l'I.C.P. entre Gabriel Ruprich-Robert et Mgr Baudrillart montre que le père a eu quelque peine à faire accepter son successeur au conseil d'administration. Si les plans de 1931 conservés aux archives de Paris (V.012/25) sont signés d'Étienne, ceux de 1933 sont à nouveau de Gabriel à qui on peut certainement attribuer l'entière paternité des bâtiments de l'Institut.

48. $\mathrm{Au}$ cours du XXe siècle, de nombreux instituts, centres de recherches et écoles complémentaires ont été créés au sein de l'I.C.P.

49. Alfred Baudrillart, Nos grandes écoles. L'Institut catholique, 1930, 130 p., p. 119.

\section{RÉSUMÉS}

Fondée en 1875 à la faveur de la loi sur la liberté de l'enseignement supérieur, l'Université, puis Institut catholique de Paris, a occupé pendant une vingtaine d'années les bâtiments du couvent des Carmes entre les rues d'Assas et de Vaugirard. En 1894, un premier édifice est construit par l'architecte des monuments historiques, Gabriel Ruprich-Robert, dans un style ogival néonormand en brique et pans de fer. Confronté à l'absence de moyens et à une Troisième République anticléricale qui met les biens religieux sous séquestre par la loi de 1905, l'Institut catholique doit attendre 1929 pour être autorisé à racheter ses immeubles et achever, en 1934, le bâtiment de la rue d'Assas avec le même Gabriel Ruprich-Robert dans le style adopté quarante ans auparavant.

Founded in 1875 in the wake of the law on Higher Education, the University then Catholic University of Paris has been housed in the monastic premises of the Carmelites in the rues d'Assas and de Vaugirard for about twenty years. In 1894 a first building is constructed of brick and iron-framed in a Gothic neo-Roman style by the architect of Historic Monuments, Gabriel Ruprich-Robert. As it has to face financial difficulties and an anticlerical Third Republic that sequesters religious goods in accordance with the 1905 Law, only in 1929 is the Catholic 
University of Paris allowed to buy back its premises and to have the rue d'Assas building completed by the same Gabriel Ruprich-Robert in the same style used forty years ago.

1875, auf Anregung des Gesetzes über die Unterrichtsfreiheit im Hochschulwesen begründet, bewohnten die Universität und dann das Pariser katholische Institut ungefähr zwanzig Jahre lang die Gebäude des Karmeliterklosters zwischen den Straßen d'Assas und Vaugirard. 1894 wurde ein erstes Gebäude von dem Staatsarchitekten Gabriel Ruprich-Robert im neonormannischen Ogivalstil aus Ziegelstein und Stahl gebaut. Konfrontiert mit Geldmangel infolge der 1905 getroffenen antiklerikalen Gesetze der Dritten Republik, insbesondere der Beschlagnahme des kirchlichen Eigentums, durfte das katholische Institut erst 1929 seine Grundstücke zurückkaufen. Erst 1934 wurde das Gebäude in der rue d'Assas beendet, von Gabriel Rupricht-Robert in dem gleichen Stil gebaut wie vierzig Jahre vorher geplant.

\section{AUTEUR}

\section{ANNE GOERGEON-LISKENNE}

Anne Georgeon-Liskenne, née en 1972, est conservateur du patrimoine au département des Archives historiques du ministère des Affaires étrangères. Elle a soutenu un DEA à l'École pratique des hautes études en 1996 sur « l'organisation professionnelle des architectes en France, en Allemagne et en Autriche entre 1850 et 1900 » puis une thèse d'École des chartes sur « les relations architecturales franco-germaniques entre 1840 et 1900 à travers les revues d'architecture de l'époque " sous la direction de Jean-Michel Leniaud. Elle a écrit des articles sur ces sujets et sur la construction des bâtiments d'archives au XIX ${ }^{\mathrm{e}}$ siècle. Adresse électronique : anne.georgeon@diplomatie.gouv.fr 